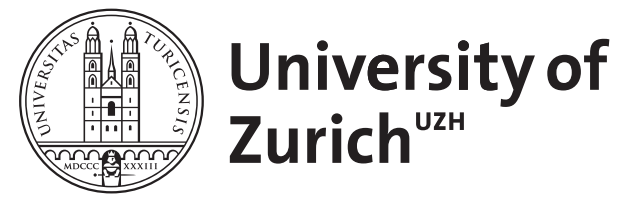

\title{
Auto(r)referentialität am Beispiel einer Collage von Kurt Schwitters
}

Schmid, Marcel

DOI: https://doi.org/10.1515/9783110400465.405

Posted at the Zurich Open Repository and Archive, University of Zurich

ZORA URL: https://doi.org/10.5167/uzh-140409

Book Section

Published Version

Originally published at:

Schmid, Marcel (2014). Auto(r)referentialität am Beispiel einer Collage von Kurt Schwitters. In: Schaffrick, Matthias; Willand, Marcus. Theorien und Praktiken der Autorschaft. Berlin: De Gruyter, 405-417. DOI: https://doi.org/10.1515/9783110400465.405 


\title{
Auto(r)referentialität am Beispiel einer Collage von Kurt Schwitters
}

\begin{abstract}
Autoreferentialität bezeichnet in der Regel eine spezifische Form der Selbstbezüglichkeit. Wenn davon ausgegangen wird, dass Texte nicht nur ihr Geschriebensein und ihre Interferenz mit Institutionen wie beispielsweise derjenigen der >Literatur « - dies wäre Autoreflexivität - reflektieren, sondern diese Reflexion auch zu ihrer Referenz machen, dann handelt es sich um einen Prozess, der von dieser spezifischen Form der Selbstbezüglichkeit charakterisiert wird. Wenn sich die Reflexion selbst zur Referenz entwickelt, dann wird die Referenz problematisch, denn die Prozesshaftigkeit der Reflexion verhindert die Stabilisierung der Referenz. Die Reflexionen des Geschriebenseins und der Interferenz bilden so eine instabile Basis, wobei eine Referenz außerhalb des `Kunstwerks`, wie sie zum Beispiel >der Autor` darstellt, für die Analyse irrelevant wird. Das heißt jedoch nicht - und das ist die Grundannahme dieses Beitrags -, dass der >Autor « verschwindet. Im Gegenteil: Weil Autoreferentialität jegliche Referenzstabilisierung subvertiert, (er)findet sich Autorität in fortwährend sich wandelnder Funktion neu. Ziel dieses Beitrags ist es zunächst, Autoreferentialität als Terminus darzulegen, um danach am Beispiel einer Collage von Kurt Schwitters die Subversion in der fortwährend sich wandelnden Funktion von Autorschaft zu untersuchen.
\end{abstract}

\section{Was heißt Autoreferentialität?}

Elektrische Gitarren und immer diese Texte, das will doch keiner hören [...]. ${ }^{1}$

Was Die Ärzte im Refrain des Liedes Junge wutentbrannt ins Mikrophon brüllen, zeigt auf bestechend einfache Weise das, was hier Autoreferentialität genannt wird: Texte, die sich selbst zu ihrer primären Referenz machen.»[...] diese Texte, das will doch keiner hören [...] «. »Diese Texte« sind genau dieselben, die im Moment vorgetragen werden. Sie schließen sich selbst mit ein. Es handelt sich um Texte, die »keiner hören « will, obwohl durch das »Hören« erst erkenntlich wird, dass sie »keiner hören « will. Der Witz liegt einerseits in der Ellipse, die zwischen »immer diese« und »Texte« ein negativ konnotiertes Adjektiv ersetzt. Andererseits liegt er auch darin, dass »diese Texte«, die »keiner hören« will, genau von

1 Die Ärzte: Junge. 
jenen gehört wird, welche die Musik kaufen und diese Texte hören wollen. Nebst diesen ironischen Selbstbezeichnungen verweist die Tatsache, dass die Liedzeile gesungen wird, auf den Modus der Übertragung. Es wird nicht nur die Semantik des Textes kontaminiert, sondern auch die Form der Übertragung. Die Semantik des Textes korrespondiert mit seiner medialen Umsetzung. Die »elektrische(n) Gitarren« setzen genau dort ein, wo im Liedtext ihre Existenz formuliert wird.

Das Beispiel zeigt einen Text, der sein Potential unablässig aus den Selbstverweisen schöpft. Dieses Verfahren hat sich mittlerweile als ein Gegenstand literaturwissenschaftlicher Forschung etabliert und wird oft mit den Termini >Autoreferentialität oder >Autopoiesis` in Verbindung gebracht. ${ }^{2}$ Die Grundlage dafür liefert die Erkenntnis, dass viele Formen von Texten nicht einfach nur Aussagen etwa über religiöse, soziale, psychische oder politische Sachverhalte treffen, sondern immer auch - und offenbar unhintergehbar - ihre eigene textuelle Verfasstheit thematisieren.

Die Literaturwissenschaft bedient sich dabei meist des systemtheoretischen Begriffs der Autopoiesis. Niklas Luhmann verwendet damit einen Begriff, der durch den Biologen Humberto Maturana geprägt wurde: "An autopoietic system is, from the point of view of its dynamics of states, a system that, while autopoietic, only generates states in autopoiesis «. ${ }^{3}$ Luhmann übersetzt und ergänzt: »Das System erzeugt sich selber. Es stellt nicht nur die eigenen Strukturen her, so wie gewisse Computer Programme für sich selber entwickeln können, sondern es ist auch auf der Ebene der Operationen autonom «. ${ }^{4}$ Äußerst wichtig für ein Verständnis von Autopoiesis ist der Verfahrenscharakter des sich selber erzeugenden Systems, denn »[...] im Vollzug reproduziert sich das System laufend selbst, verfährt somit immer selbstreferentiell«. ${ }^{5}$ Damit ist das Verfahren jedoch von einer »zirkulären Tautologie bedroht«, die durch die laufende Selbsterzeugung selbst erzeugt ist und »die letztlich den Stillstand des Systems [...] zur Folge hätte«. ${ }^{6}$ Deshalb gibt es notwendigerweise immer ein Wechselspiel zwischen

2 Zur Autoreferentialität vgl. u. a. Seelhorst: Autoreferentialität, S. 29-36; Schwanitz: Systemtheorie, S. 55-101.

3 Maturana: Autopoiesis, S. 22.

4 Luhmann: Einführung, S. 110.

5 Lickhardt: Selbstreferenz/Fremdreferenz, S. 369. In Anlehnung an die Ausführungen Luhmanns in Soziale Systeme (vgl. Luhmann: Soziale Systeme, S. 59, 604) weist Maren Lickhardt auf den Verfahrenscharakter des Wechselspiels zwischen Selbst- und Fremdreferenz hin.

6 Lickhardt: Selbstreferenz/Fremdreferenz, S. 369. 
Selbstreferenz und Fremdreferenz, um das System als Verfahren zu erhalten. ${ }^{7}$ Unter diesen hier nur kurz skizzierten Prämissen fand Autopoiesis Einzug in die Literaturwissenschaft. Dabei herrscht weder Einigkeit, was Autopoiesis bezüglich der Literatur und insbesondere bezüglich der Semantik heißen soll, ${ }^{8}$ noch existiert dazu eine konzise Untersuchung zum Verhältnis von Autopoiesis und Autoreferentialität. ${ }^{9}$ So schlage ich vor, Autopoiesis vereinfachend als das Verfahren der kontinuierlichen Selbsterzeugung zu bezeichnen. Autoreferentialität würde dann auf die Referenzproblematik der Selbsterzeugung fokussieren. Wenn nun um nochmals auf das Beispiel Die Ärzte zurückzukommen - »diese Texte« sich selber mit einschließen, dann lässt dies Rückschlüsse auf die Referenzproblematik zu. »Diese Texte« verweisen auf eine Referenz außerhalb des Textes - also auf eine Fremdreferenz - und auf eine Selbstreferenz, denn sie schließen auch die Buchstabenfolge »d/i/e/s/e T/e/x/t/e« ein. Es ist nun offensichtlich, dass daraus ein semantisches >Problem außerhalb des Textes referieren, sondern auf sich selbst, dann wird der Aussagegehalt problematisch. Was heißt »diese Texte«? Allerdings resultiert aus der Unmöglichkeit der Beantwortung der Frage auch ein Potential, nämlich das Nachdenken über Sprache und ihren Referenzbezug.

Wenn durch die Prozesshaftigkeit der Reflexion des Geschriebenseins jegliche Referenz destabilisiert wird, dann dürfte damit auch die Referenz außerhalb des >Kunstwerks`, wie sie zum Beispiel >der Autor`darstellt, für die Analyse irrelevant sein. Die Kenntnis, dass die Worte »diese Texte« von Die Ärzte stammen, scheint zumindest auf den ersten Blick wenig zur Lösung des Referenzproblems beizutragen. Wenn davon ausgegangen wird, dass Autoreferentialität einen Prozess der Referenz-Entortung charakterisiert, dann ist >der Autor ‘ als Kategorie unsicher geworden, weil er als stabilisierende Referenz nicht mehr taugt. Das heißt jedoch nicht, dass >Autorschaft ` verschwindet. Im Gegenteil: Weil Autoreferentialität jegliche Referenzstabilisierung subvertiert, (er)findet sich Autorität in fortwährend sich wandelnder Funktion neu. Die Selbstbezeichnung durch »diese

7 Vgl. Lickhardt: Selbstreferenz/Fremdreferenz, S. 370; Luhmann: Einführung, S. 82-83. Luhmann bezeichnet dabei ১Selbstreferenz als dasjenige, »was im System läuft« und `Fremdreferenz« als dasjenige, was die »internen und externen [...] Systemzustände« betrifft.

8 So könnte man darüber streiten, ob selbstbezügliche Zeichen `keine` Zeichen mehr sind. Vgl. Christoph Bode: Ästhetik der Ambiguität, S. 377: "Zeichen, die selbstbezüglich werden, sind im gleichen Grade, wie dies gelingt - keine Zeichen im herkömmlichen Sinne mehr, sie bedeuten nur sich selbst, wollen auf gar nichts anderes verweisen, versuchen nur zu sein«.

9 Die Ausnahme bilden zwei (systemtheoretische) Texte von David E. Wellbery, die sich dem Thema auch semiologisch annähern. Wellbery: Retrait/Re-entry, S. 194-207; Wellbery: Das Gedicht, S. 366-383. 
Texte« lässt sich nicht durch Die Ärzte stabilisieren - darum geht es in der Untersuchung von Autoreferenzialität auch in keiner Weise -, sondern Texte entwerfen Autorschaft als Prozess mit. Der ironische Selbstkommentar »[...] und immer diese Texte [...] « sagt deshalb auch etwas über die Inszenierung von Autorschaft aus. Somit werden Die Ärzte durch ihre Texte entwickelt. Die Entwicklung von Autorschaft als Prozess hat vor allem in der Avantgarde um 1900 seine interessanten Vorläufer. Diese sind besonders anschaulich, weil oft kein >direkter $\mathrm{Zu}$ gang über die Kategorie des Verstehens gefunden werden kann. Somit wird die Fokussierung auf formale Aspekte und auf das Verfahren des Kunstwerks erleichtert. Bezüglich des Surrealismus hat beispielsweise Walter Benjamin treffend formuliert, wo dessen Prinzip liege, nämlich dort: »[...],[wo]die Sprache nur sie selbst [ist], wo Laut und Bild und Bild und Laut mit automatischer Exaktheit derart glücklich ineinandergriffen, dass für den Groschen `Sinn` kein Spalt mehr übrig blieb «. ${ }^{10}$ Der sinn « bleibt weg, so dass - nach Luhmann (und mit Novalis gesprochen) -: »[d]ie Sprache nur mit sich selbst [spielt] «. ${ }^{11}$

Die im Folgenden vorgestellte Collage von Kurt Schwitters ist ein Beispiel aus dem Dadaismus und soll zeigen, dass Autorschaft als Prozess im >Taschenspielertrick « ausgestellt wird. Die titellose Collage, die 1945 bis 1947 entstanden ist, ${ }^{12}$ bietet eine gute Basis, um über Autorschaft und die brüchige Grenze zwischen Autoreferentialität und Autorreferentialität nachzudenken. Dabei geht es weder darum, eine umfassende, semiologische Theorie von Autoreferenzialität zu entwickeln, noch darum, diesen Terminus zu historisieren. Vielmehr soll in Anlehnung an die Tradition des close readings Autoreferenzialität gerade in Verbindung mit der Autorschaftsthematik als ein Entstehendes skizziert werden, ${ }^{13}$ welches notwendig keiner klar formulierbaren Theorie entspricht.

10 Benjamin: Der Sürrealismus, S. 150.

11 Luhmann: Gesellschaft, S. 994.

12 Schwitters: Ohne Titel, S. 287.

13 Dabei ist durchaus an dekonstruktive Spielarten dieses close reading zu denken, wie sie etwa Paul de Man in Allegorien des Lesens am Beispiel von Rilke, Proust oder Nietzsche exemplifiziert. 


\section{August Heinrich Kerndörffer}

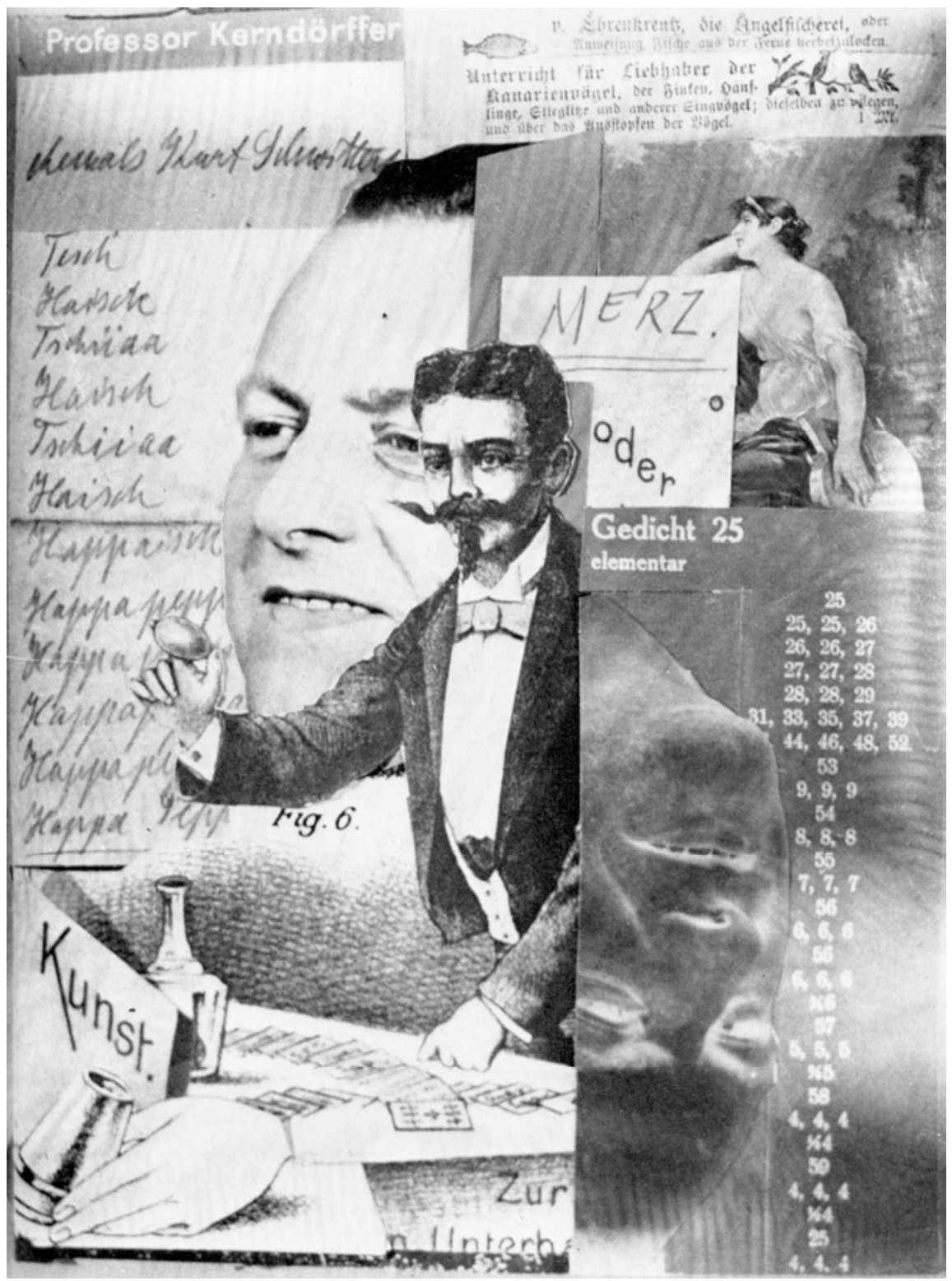

Abb. 1: Ohne Titel. 1945-47. Collage. Privatbesitz 
Dort, wo wir zu lesen beginnen würden, oben links, zeigt sich ein Name, der, anders als es zumindest Eigennamen meistens suggerieren, gerade nicht Gewähr für einen konkreten Quellenhinweis geben kann. »Kern-dörffer« sozusagen die Selbstbezeichnung in nuce zu Beginn ist nicht mehr Kurt Schwitters, sondern ein doppelter (raumzeitlicher) Witz. Erstens ist dieser - rhetorisch - als ein hysteron proteron zu verstehen, was die zeitliche Verschiebung des Autornamens zu einem mutmaßlichen Taschenspieler und Trivialisten zeigt, der hundert Jahre vor Schwitters gelebt hatte und zweitens ist er zugleich räumlich, denn die Verbindung zum »Kern«, zum Zentrum der Collage mit der Figur des Zauberers in der Mitte ist augenfällig.

Heinrich August Kerndörffer (1769-1846) ist ein Autor, der zwar Ende des 18. Jahrhunderts viel gelesen, von der heutigen Forschung jedoch fast gänzlich vergessen wurde. ${ }^{14}$ Dabei ist nicht nur die Tatsache bemerkenswert, dass Kerndörffer als akademischer Lehrer an der Universität Leipzig Heinrich von Kleist - den Stotterer - in der Deklamationskunst unterrichtete. ${ }^{15}$ Der Umfang der Kerndörffschen Publikationsliste und die Breite seiner Interessengebiete wären alleine schon eine Untersuchung wert.

So ist der Lehrer Kleists Herausgeber eines Modemagazins ( Leipziger ModeMagazin, 1801-1803) ${ }^{16}$ und Verfasser mehrerer Deklamationshandbücher, darunter auch das dreibändige Handbuch der Deklamation (Leipzig 1813-15). ${ }^{17}$ Zudem finden sich 21 pädagogische und serbauliche`Schriften, die sich vor allem an Kinder (zum Beispiel Kleines Fabelbuch für Kinder edler Erziehung, oder Leben der Tugend und sittlichen Klugheit in Unterhaltung eines Lehrers mit seinen Zöglingen , Leipzig 1802) ${ }^{18}$ und insbesondere an die liebende Jugend richtete (zum Beispiel Über die Liebe, allen liebenden Jünglingen und Mädchen gewidmet , Leipzig 1794). ${ }^{19}$ Doch Kerndörffer war den Zeitgenossen vor allem für seine Trivialromane und kompilatorischen Schauermärchenbände bekannt, die zwar hohe Auflagenzahlen erreichten, aber schon von der zeitgenössischen Kritik als `belanglos` taxiert

14 Es gibt tatsächlich nur sehr wenige wissenschaftliche Arbeiten oder Einträge in Lexika zu Kerndörffer. Die Informationen zu ihm beruhen in erster Linie auf folgenden Texten: Meyer-Kalkus: Kleist und H.A. Kerndörffer, S. 55-88; Killy: Literatur Lexikon. Bd. 6, S. 299-300; Kosch: Deutsches Literatur-Lexikon, Bd. 8, S. 1085; Weidemeier: Kerndörffer.

15 Meyer-Kalkus: Kleist und H.A. Kerndörffer, S. 55, 62.

16 Weidemeier: Kerndörffer, S. 55.

17 Ebd., S. 53.

18 Ebd., S. 54.

19 Ebd., S. 55. 
wurden. ${ }^{20}$ Kerndörffers Textkonvolut beinhaltet somit eine beeindruckende Vielfalt an Gattungen. Dabei machen besonders die Abenteuerromane einen ziemlich abgedroschenen Eindruck. Dies zeigt sich schon am Beispiel des in mehreren Auflagen 1821 erschienenen Abenteuerromans Lorenzo, der kluge Mann im Walde, oder das Banditenmärchen, in dem ein zu Unrecht in den Wald verbannter "Staatsmann « mit dem Übernamen »Lorenzo« gegen die "Verschwörung vom schwarzen Bunde« des Staates Ysamo kämpft und selbstverständlich rehabilitiert wird. ${ }^{21}$ Nach demselben Muster sind die meisten weiteren Räuber- und Abenteuerromane gestrickt: Ein Ausgestoßener, ein Außenseiter kämpft in einer wilden Welt (Wald, Gebirge, etc.) gegen einen bösartigen Feind, welcher nicht nur den Helden aus der Umgebung des Staates oder des Hofes intrigant und / oder gewaltsam entfernt hatte, sondern dadurch auch die (Hof-)Staatssicherheit gefährdet. ${ }^{22}$

\section{Der collagierte Zaubertrick}

In Zusammenhang mit der Collage von Kurt Schwitters sind die Lehrbücher für Zauberei und Kryptographie besonders interessant. Einen kleinen, (wohl gänzlich unfreiwillig) selbstbezüglichen Witz liefert ein Titel zur Kryptographie:

Leicht fassliche Anleitung zur Kryptographie oder den verschiedenen Arten der geheimen Schreibekunst, in Verbindung mit der Stenographie und Tachygraphie oder Geschwindschreibekunst und ihrer Anwendung für die mannichfaltigen Verhältnisse und Angelegenheiten des Staatslebens neuerer Zeit (Leipzig 1835). ${ }^{23}$

Es scheint hier der gesamte Texttitel gegen die ersten drei Worte »Leicht fassliche Anleitung « angelegt zu sein. Genau dieser in sich selbst leicht kryptische Titel ist symptomatisch für die Figur Kerndörffers. Er verfügt über ein `Leben` und `Werk

20 Z. B. in der Neuen allgemeinen Bibliothek, Siehe: Weidemeier: Kerndörffer, S. 80.

21 Weidemeier: Kerndörffer, S. 79-80.

22 Zudem nennenswerte, weil auch sprechende Titel sind zum Beispiel der in zwei Bänden erschienene Ullo, der Barde des Gebirges oder Die Schreckgeister in den Klüften des Chilo-Felsens von 1806 (vgl. Kosch: Deutsches Literatur-Lexikon, Bd. 8, S. 1085; Weidemeier: Kerndörffer, S. 81) oder Dämonio, der umherwandelnde Unhold, oder Das verschleierte Bild auf den Höhen des Schreckhorns. Eine abenteuerliche Erzählung (Leipzig 1807) (vgl. Kosch: Deutsches Literatur-Lexikon, Bd. 8, S. 1085; Weidemeier: Kerndörffer, S. 83).

23 Es erweist sich als schwierig, dieses Werk bibliographisch einzuordnen. So wird es zum Beispiel in einem umfassenden Band zur Stenographie von 1895 erwähnt. Faulmann: Stenographie, S. 98 . 
das genau diese beiden Kategorien unsicher werden lässt. Angesichts der Breite seiner Tätigkeiten und seiner schriftlichen Arbeit stellt sich die Frage, ob hier ein (literarischer) Taschenspieler am Werk ist. Im Zentrum der Collage findet sich ein Taschenspieler, der etwas mit der zu Beginn schon formulierten These zu tun hat: Autorschaft wird als Prozess im `Taschenspielertrickı ausgestellt. Es ist eine Figur abgebildet, die ein Ei in der Hand hält und über einen Tisch mit einer Flasche und ausgelegten Spielkarten gebeugt ist. Im Vordergrund ist zudem eine weitere Hand zu sehen, die einen Becher hält. Um nochmals auf die Behauptung zurückzukommen, dass der oben links abgebildete Name Kerndörffer etwas mit der Figur im Zentrum zu tun habe, muss Folgendes vorausgeschickt werden: Kerndörffer machte sich nebst den oben erwähnten Bereichen zudem einen Namen als Verfasser und Herausgeber mehrerer Zauberbücher.

So finden sich Titel wie zum Beispiel Der kleine Taschenspieler und Magiker, oder Anweisung, verschiedene Taschenspielerkünste und magische Täuschungen mit wenigen Kosten nachzumachen. Zur Unterhaltung und Belehrung für manches unerklärbar Scheinende (Leipzig 1801) ${ }^{24}$ und Carlo Boscos Zauberkabinet, oder das Ganze der Taschenspielerkunst, welches bis 189921 Auflagen erreichte. ${ }^{25}$ Gerade letztgenanntes offenbar weitverbreitetes Werk birgt die Unsicherheit, ob Kerndörffer, der als Herausgeber erscheint, nicht doch auch Autor des Werks gewesen ist. Ob die Figur in der Mitte »Carlo Bosco« oder sonst einen prototypischen Zauberer darstellt, kann nicht abschließend geklärt werden. Entscheidend ist hier, wie Referenzbildung und die Unsicherheiten bezüglich dieser Referenzbildung auf der Ebene der Autorschaft konvergieren. Die Referenzen sind in vielerlei Hinsicht zweifelhaft. Das Phänomen `Kerndörffer ‘ führt gerade nicht in die Würde und (wissenschaftliche) >Klarheit`, die sich im ersten Wort der Collage manifestieren: »Professor «. >Kerndörffer« verschiebt sich vielmehr zum Zauberer im Zentrum in doppelter Hinsicht: als Verfasser magischer Schriften und als Figur des Zauberers selbst. In beiden Fällen bleiben Kerndörffer und Bosco komplett unsichere Instanzen. Es scheint sich, wie es auf Italienisch der Name »Bosco« schon sagt, nicht ein statisches Zentrum, sondern - auf Deutsch - ein »Wald«, ein undurchsichtiges Geflecht zu eröffnen. So führt das Zentrum, in seiner Punktualität entäußert und einer rhizomatischen Struktur unterworfen, in die Paradoxie des `Zentrums s ohne Zentrum. Und das ist zugleich der Taschenspielertrick und - auf Basis der Autorschaft - eine entscheidende Problematik von Referenz: Es gibt oft starke Hinweise, die auf den Pfad der Verortung des

24 Weidemeier: Kerndörffer, S. 54.

25 Ebd. 
>Kunstwerks` führen, also Anreize schaffen, die der Referenzbildung dienen sollen, dabei jedoch nur ins Leere laufen lassen. Die Bedingung der Möglichkeit zur Referenzbildung ist selbst Grund, diese zu verhindern. Um diesen Prozess darzulegen, lohnt sich ein Blick auf die Fotographie Schwitters in der Collage.

\section{Die Bilder Schwitters}

Die Fotographie stellt zweifelsfrei Schwitters selbst dar. Es gibt nebst der >Unterschrift, die hier - links oben - paradoxerweise als Überschrift fungiert, wohl kaum stärker verifizierende Hinweise auf die sautoritäre` Referenz: das Erscheinen des Autors in Text und Bild. Beide, Bild und Unterschrift, stecken nicht nur das Territorium des geistigen Eigentums ab, sondern werden gerne als Folien von Sinnstabilisierungen benutzt. Schon die Tatsache, dass diese Referenzbildung hier im wahrsten Sinn des Wortes in-szeniert ist, disqualifiziert jeglichen Versuch, zum Zentrum der Collage vorzudringen. Jede Inszenierung entwirft selbst ein Bild und kann nicht selbst Ursprung sein. Die Tatsache, dass die Inszenierung der Selbst-Referenz auf dem Hintergrund einer Collage geschieht, ironisiert die Inszenierung zusätzlich.

Eine Collage vereint aus dem Zusammenhang gerissene Versatzstücke wie beispielsweise aus Text-, Bild- und Holzelementen und ordnet sie neu. Auf diese Weise wird das Durchbrechen einer sequentiellen, logischen Ordnung präsentiert, was wiederum eine Fokussierung auf formale Aspekte erleichtert. So wirkt sich auch die Autorität des Bildes Schwitters und seiner Unterschrift destabilisierend aus. Der Autor übernimmt keine Referenzfunktion, obwohl er und sein Schaffen immerfort inszeniert werden. Dies zeigt sich nicht nur an den Fotographien und an den Unterschriften. Bei den schwierig zu entziffernden handgeschriebenen Wörtern auf der linken Seite der Collage handelt es sich um einen Auszug aus dem lautmalerischen Gedicht Nießscherzo aus dem Jahre 1936. ${ }^{26} \mathrm{Al}-$ lerdings scheint das Gedicht für die Collage verändert worden zu sein. Die Reihenfolge der Lautzeilen »Tesch«, »Haisch«, »Tschiiaa«, »Haisch«, »Happaisch «, usw. stimmt nicht mit der Reihenfolge in Friedhelms Lachs Gesamtausgabe überein. ${ }^{27}$ Das handschriftliche Nießscherzo mit der klar sichtbaren Fotographie

26 Wie Sigrid Franz richtig bemerkt, muss die Datierung in der von Friedhelm Lach herausgegebenen umfangreichen Werksausgabe korrigiert werden. Das Gedicht - so geht es aus einem Brief an Annie Müller-Widmann hervor - ist erst im Herbst 1937 entstanden. Franz: Merz-Ästhetik, S. 125.

27 Schwitters: Nießscherzo, S. 244-245. 
Schwitters findet seine (subversive) Entsprechung auf der Seite rechts unten, nämlich dort, wo das Foto Schwitters verkehrt wird und dies gleich in doppelter Hinsicht: Einerseits ist Schwitters um die horizontale und vertikale Achse gedreht, andererseits wird er fotonegativ dargestellt, das heißt: hell wird dunkel und umgekehrt. Diese fiktiven Achsen schneiden sich in der Mitte der Collage, im Kopfbereich der zaubernden Figur. Betrachtet man die Farbgebung, so passt das Fotonegativ auf die Seite rechts unten schon nur aufgrund der >Dunkelheit, als müsste sie auf die >Nachtseite unserer (lese)gewohnten Hierarchisierung aufmerksam machen: rechts unten, neben der 'Zahlenmagier des Gedichts 25 elementar, ${ }^{28}$ das sich drohend zum Kreuz formiert. Die $>$ Nachtseite der Schrift (»vérité noire de l'écriture«) ist nach Roland Barthes jene ১Seite`, die nicht der klaren Kommunikation dient, sondern verbirgt und für die Unwissenden sich hin zur Kryptographie entwickelt. ${ }^{29}$ Auf dieser Seite wird Kurt Schwitters als klare Referenz tatsächlich gewesen sein, »ehemals«, wie schon ganz oben in der Collage rangekündigt«.

Beim Gedicht 25 elementar handelt es sich um ein lyrisches Werk Schwitters, welches schon um 1926 entstanden ist. Bemerkenswert ist also, dass Schwitters nicht nur seine Person in Verbindung mit Kerndörffer darstellt, sondern auch eigene Werke um die Figur des Zauberers anordnet. Dass gerade diese Gedichte die beiden Fotographien Schwitters umrahmen, scheint nicht zufällig. Der onomatopoetischen Seite (Nießscherzo) wird die Zahlenpoesie gegenübergestellt. Die bei Zahlenreihen übliche Logik ist allerdings nicht feststellbar. Dennoch verführt gerade das Gedicht zum Herstellen solcher Logiken, wie zum Beispiel in Zahlen, die für Buchstaben stehen, in Zahlen, die addiert, subtrahiert, multipliziert oder dividiert einen Sinn ergeben sollten, es aber in keiner Weise tun. Vielleicht liegt darin auch der Grund, wieso das Gedicht 25 elementar auf der >dunklen Seiter liegt. Selbst Zahlen, die eigentlich einer streng logischen Funktionsweise folgen, sind hier in ihrer Kombination undurchschaubar, sodass auch sie nicht an das `Licht` führen. Was sich somit hier deutlich zeigt: Trotz und gerade wegen dem

28 Schwitters: Gedicht 25 elemtar, S. 204.

29 Roland Barthes zeigt im Kapitel Verbergen in seinem Essay Variations sur l'écriture auf, dass die (zeitgenössischen) Sprachforscher sich zu sehr auf die kommunikative Funktion der Sprache stützen. Nach Barthes hat die Sprache auch eine verbergende Funktion. Dies zeigt sich zum Beispiel dort, wo sich die Sprache aus religiösen und sozialen Gründen nicht allen verfügbar macht. Dabei geht Barthes in erster Linie auf die immer auch mitzudenkende nicht-kommunikative Funktion der Schrift ein. Als einleuchtendes Beispiel erwähnt er das Manuskript, das umso persönlicher »gilt, je schwieriger sie [Schrift] zu lesen ist«. Barthes: Variations sur l'écriture, S. 2327. 
penetranten Verweis auf den Autor und seine Werke, werden der Autor und sein Werk immerfort subvertiert.

\section{Zusammenfassung und Ausblick}

Der Prozess in dieser Collage ist das gleichzeitige Verdecken und Enthüllen: Fotopositiv - Fotonegativ, Referenz - Referenz, Schwitters - Kerndörffer, Nießscherzo - Gedicht 25 elementar. Diese Konstallation dynamisiert die nur scheinbar stabilen Begebenheiten und führt dazu, dass der Autor als stabile Entität unmöglich ist, aber gleichzeitig durch die Inszenierung der Autorität als Prozess ermöglicht wird. >Taschenspielertrick « wurde dieser Prozess hier genannt, weil er nur verblüffend das (in sich selbst) unsichere Resultat eines Prozesses zum Vorschein bringt, eines Prozesses, der paradoxerweise die Verflüchtigung der Autorreferenz in der Fokussierung auf die Autorreferenz initiiert: die Entfokussierung oder Destabilisierung erfolgt durch die Fokussierung oder die mögliche Stabilisierung. Der >Taschenspieler` in der Mitte steht für einen Prozess, an dem er gleich selbst beteiligt ist: Indem er in seiner gesamten materiellen Präsenz sich als Referenz auslöscht. Es ist die gleichzeitige Möglichkeit zur Referenzbildung und die Subversion dieser Möglichkeit oder anders gesagt: Das stetige Verweisen auf die Referenz der Autorschaft ist hier Taschenspielertrick.

Es ist schwierig, die Ebene der hier untersuchten Collage zu verlassen und den anfänglich erwähnten Bezug zwischen Autorreferentialität und Autoreferentialität zu verallgemeinern. Autoreferentialität heißt nicht, dass Autorschaft eine Rolle spielen muss. Vielmehr eröffnen sich am Beispiel der Autorschaft die wohl interessantesten und auch anschaulichsten Spielarten von Autoreferentialität. Im Zusammenhang mit der Collage sind zwei Punkte für eine zukünftige Forschung zentral: Es gibt gerade zu Schwitters »Merz«-Begriff zahlreiche fundierte Untersuchungen, die daraus - manchmal im Vergleich mit weiteren Dadaisten ein ästhetisches Prinzip für die »Merzkunst « selbst und / oder die gesamte Avantgarde abzuleiten versuchen. ${ }^{30}$ Vielleicht ist es ergiebiger, die Kunst aus ihrem hermetischen Dasein als spezifische Avantgarde- oder Dadakunst zu lösen und

30 Siehe dazu: Franz: Merz-Ästhetik; Fux: Merzwelt; Reumkens: Allotria ad absurdum; Winkelmann: Abstraktion. Die Ausnahme bilden hier Hans-Jürgen Hereths rezeptionsästhetisch orientierter Text Die Rezeptions- und Wirkungsgeschichte von Kurt Schwitters, dargestell anhand seines Gedichts >An Anna Blumer und vor allem Beatrix Nobis lesenswerte Studie zu Kurt Schwitters und die Romantische Ironie. Nobis legt dar, wie Schwitters Schaffen in der Tradition der Romantischen Ironie steht und als philosophisch-ästhetisches Problem zu betrachten ist. 
vermehrt in zwei Bereiche zu blicken: Zum einen betrifft dies den diskursgeschichtlichen Kontext. Es ist wohl kaum ein Zufall, dass die Dadaisten die Kunst in einer Zeit zu reformieren versuchten, die angelehnt an die verschiedenen »Lebensreformbewegungen " auch schon "Laboratorium der Moderne" genannt wurde. ${ }^{31}$

Zweitens lässt sich auch eine komplexe literaturtheoretische Beobachtung wie Autoreferentialität zweifelsohne gerade an Texten und Kunstwerken jenseits des literarischen Höhenkamms sehr anschaulich darlegen. Deshalb sollte hier auch klar geworden sein, dass Autoreferentialität nicht nur als Avantgarde-Collage fruchtbar wird, sondern auch - wie anfänglich gezeigt - als trivialer Spaß, mit dem nicht nur angehende Doktoren, sondern auch Die Ärzte gerne operieren.

\section{Bibliographie}

Barthes, Roland: Variations sur l'écriture. Variationen über die Schrift. Französisch - Deutsch. Mainz 2006.

Benjamin, Walter: „Der Sürrealismus. Die letzte Momentaufnahme der europäischen Intelligenz«. In: Opitz, Michael (Hrsg.): Walter Benjamin. Ein Lesebuch. Frankfurt a. M. 1996, S. 149-164.

Bode, Christoph: Ästhetik der Ambiguität. Zur Funktion und Bedeutung von Mehrdeutigkeiten in der Literatur der Moderne. Tübingen 1988.

Buchholz, Kai / Rita Latocha / Hilke Peckmann / Klaus Wolbert (Hrsg.): Die Lebensreform. Entwürfe zur Neugestaltung von Leben und Kunst um 1900, 2 Bde. Darmstadt 2001.

De Man, Paul: Allegorien des Lesens. Frankfurt a. M. 1988.

Die Ärzte, »Junge«. In: Jazz ist anders (Album). Berlin 2007, Stück Nr. 6.

Faulmann, Karl: Geschichte und Litteratur der Stenographie. Wien 1895.

Franz, Sigrid: Kurt Schwitters' Merz-Ästhetik im Spannungsfeld der Künste. Freiburg i. Br. u. a. 2009.

Fux, Evelyn: Schnitt durch die verkehrte Merzwelt. Konzeptionen des Narrativen in der Prosa von Kurt Schwitters. Berlin 2007.

Hereth, Hans-Jürgen: Die Rezeptions- und Wirkungsgeschichte von Kurt Schwitters, dargestellt anhand seines Gedichts sAn Anna Blumer. Frankfurt a. M. 1996.

Killy, Walther (Hrsg.): Literatur Lexikon. Autoren und Werke deutscher Sprache, Bd. 6. Gütersloh / München 1990, S. 299-300.

31 Umfassende Arbeiten zur Lebensreformbewegung, welche auch die jeweiligen Kunstrichtungen prominent behandeln - ohne jedoch genauer auf die Dadaisten einzugehen - gibt es einige. Die zwei wohl wichtigsten sind einerseits das von Kai Buchholz, Rita Latocha, Hilke Peckmann und Klaus Wolbert herausgegebene, zweibändige Die Lebensreform und andererseits das Handbuch der Deutschen Reformbewegungen 1880-1933, herausgegeben von Diethard Krebs und Jürgen Reulecke. 
Krebs, Diethard / Jürgen Reulecke (Hrsg.): Handbuch der Deutschen Reformbewegungen 18801933. Wuppertal 1998.

Lach, Friedhelm (Hrsg.): Kurt Schwitters. Das literarische Werk, Bd. 1 (Lyrik). Köln 1973.

Lickhardt, Maren: „Selbstreferenz/Fremdreferenz - Joseph Roth«. In: Werber, Niels (Hrsg.): Systemtheoretische Literaturwissenschaft. Begriffe - Methoden - Anwendungen. Berlin / New York 2011, S. 363-372.

Luhmann, Niklas: Soziale Systeme. Grundriß einer allgemeinen Theorie. Frankfurt a. M. 1987. Luhmann, Niklas: Die Wissenschaft der Gesellschaft. Frankfurt a. M. 1992.

Luhmann, Niklas: Die Gesellschaft der Gesellschaft, 2 Bde. Frankfurt a. M. 1998.

Luhmann, Niklas: Einführung in die Systemtheorie. Vorlesungsskript. Hrsg. v. Dirk Baecker. Heidelberg 2009.

Maturana, Humberto R.: »Autopoiesis«. In: Zeleny, Milan (Hrsg.): Autopoiesis. A Theory of Living Organization. New York / Oxford 1981, S. 21-33.

Meyer-Kalkus, Reinhart: »Heinrich von Kleist und Heinrich August Kerndörffer. Zur Poetik von Vorlesen und Deklamation«. In: Blamberger, Günter (Hrsg.): Kleist-Jahrbuch 2001. Berlin 2001, S. 55-88.

Nobis, Beatrix: Kurt Schwitters und die romantische Ironie. Ein Beitrag zur Deutung des MerzKunstbegriffes. Alfter 1993.

Reumkens, Noël: Allotria ad absurdum. Schwitters' Merzlyrik als Resonanzdimension. Bielefeld 2007.

Rupp, Heinz / Carl Ludwig Lang (Hrsg.): Wilhelm Kosch. Deutsches Literatur-Lexikon. Biographisch-bibliographisches Handbuch. 3. Aufl. Bd. 8. Bern 1981, S. 1085.

Schwanitz, Dietrich: Systemtheorie und Literatur. Ein neues Paradigma. Opladen 1990.

Schwitters, Kurt: „Gedicht 25 elementar«. In: Lach (Hrsg.): Kurt Schwitters, S. 204.

Schwitters, Kurt: »Nießscherzo. Das Ganze niesen«. In: Lach (Hrsg.): Kurt Schwitters, S. 244245.

Schwitters, Kurt: »Ohne Titel (Collage)«. In: Lach (Hrsg.): Kurt Schwitters, S. 287.

Seelhorst, Jörg: Autoreferentialität und Transformation. Tübingen / Basel 2003.

Weidemeier, Hartmut: Heinrich August Kerndörffer. Untersuchungen zum Trivialroman der Goethezeit. Bonn 1967.

Wellbery, David E.: »Das Gedicht. Zwischen Literatursemiotik und Systemtheorie«. In: Fohrmann, Jürgen / Harro Müller (Hrsg.): Systemtheorie in der Literatur. Frankfurt a. M. 1996, S. 366-383.

Wellbery, David E.: »Retrait/Re-entry. Zur poststrukturalistischen Metapherndiskussion«. In: Neumann, Gerhard (Hrsg.): Poststrukturalismus. Herausforderung an die Literaturwissenschaft. Stuttgart / Weimar 1997, S. 194-207.

Winkelmann, Judith: „Abstraktion als stilbildendes Prinzip in der Lyrik von Hans Arp und Kurt Schwitters«. In: Bochumer Schriften zur deutschen Literatur, Bd. 47. Hrsg. v. Martin Bollacher / Hans-Georg Kempner / Uwe-K. Ketelsen / Paul Gerhard Klussmann. Frankfurt a. M. 1995. 
Bereitgestellt von | UZH Hauptbibliothek / Zentralbibliothek Zürich Angemeldet Heruntergeladen am | 06.10.17 12:57 